\title{
On Global Solutions for Non-Linear Hamiltonian Evolution Equations
}

\author{
J. Marsden * \\ Department of Physics, University of California, Berkeley, California
}

Received September 11, 1972

\begin{abstract}
It is shown that partial differential equations of Hamiltonian type admit global solutions in time if (a) the initial data is near equilibrium (or the coupling constant is small) (b) the linear terms have positive energy and (c) the non-linear terms are smooth functions in the topology of the linearized energy norm. The non-linear terms need not have positive energy. The result is applied to non-linear wave equations in which the interaction energy is not necessarily positive.
\end{abstract}

\section{Introduction}

Our purpose is to give a simple technique for proving global existence of solutions for Hamiltonian systems in which the energy is not necessarily positive. Despite the trivial appearance of the theorem, it is applicable to non-linear wave equations in which case we can remove the positivity assumption on the energy which is commonly made. While our results are not as deep as those of other authors; cf. Chadam [1], they have the advantage of extreme simplicity. The methods will be applied elsewhere to study global solutions of the Einstein equations in general relativity cf. [3].

\section{Abstract Theorem}

To avoid a lot of notation concerning symplectic structures, we shall just introduce Hamiltonian systems in a special form suitable for our present purposes cf. [2].

Let $\mathrm{H}$ be a (real) Hilbert space and $A$ an (unbounded) skew adjoint operator on $\mathbb{H}$. Thus $A$ generates a one parameter group of isometries on $\mathbb{H}$. Let $V: \mathbb{H} \rightarrow \mathbb{R}$ be a smooth function and suppose:

$$
V(0)=0, D V(0)=0, D^{2} V(0)=0
$$

where $D V(0)$ is the derivative of $V$ at 0 . (Actually we require only $V$ defined on $D_{A}$, the domain of $A$.)

$\star$ Partially supported by NSF Grant GP 15735 . 
Suppose $J: \mathbb{H} \rightarrow \mathbb{H}$ is a smooth map such that the following hold:

(a) $D V(u) \cdot A v=-\langle J u, v\rangle$ for $u \in \mathbb{H}, v \in D_{A}$ and

(b) $D V(u) \cdot J u=0$.

As is simple and well-known (Segal [4]), the non-linear operator $X(u)=A \cdot u+J(u)$ generates a local non-linear flow; i.e. classical solutions in $D_{A}$ of

$$
\frac{d u}{d t}=X(u), u(0) \in D_{A} \text { prescribed }
$$

exist for small $t$-intervals and are unique. Moreover, it is easy to check that the energy

$$
H(u)=\frac{1}{2}\langle u, u\rangle+V(u)
$$

is conserned by these solutions.

Theorem. Under the above assumptions, there is an $\varepsilon>0$ such that if $\left\|u_{0}\right\|<\varepsilon$, the solution of (1) is defined and is unique for all $t \in \mathbb{R}$. Moreover, $u(t)=O(1)$ for all $t \in \mathbb{R}$.

Proof. Since $H(u)$ has a non-degenerate critical point at 0 and $D^{2} H(0)$ is the inner product on $\mathbb{H}$, it follows from Taylor's theorem that on some neighborhood of $0 \in \mathbb{H}$ we have $M_{1}\|u\|^{2} \leqq H(u) \leqq M_{2}\|u\|^{2}$ for constants $M_{1}$ and $M_{2}$. Thus by conservation of energy, the origin is stable, so an integral curve remains a priori in a small neighborhood of 0 . The local existence theory then gives the result.

Q.E.D.

\section{Application}

Consider on $\mathbb{R}^{n}$ the non-linear wave equation

$$
\frac{\hat{c}^{2} \phi}{\hat{c} t^{2}}=\nabla^{2} \phi-m^{2} \phi-\alpha \phi^{p}, p \text { an integer } \geqq 2, \quad \alpha \in \mathbb{R}, m \geqq 0
$$

or

$$
\frac{d}{d t}\left(\begin{array}{l}
\phi \\
\dot{\phi}
\end{array}\right)=A\left(\begin{array}{l}
\phi \\
\dot{\phi}
\end{array}\right)+J\left(\begin{array}{l}
\phi \\
\dot{\phi}
\end{array}\right)
$$

where $A=\left(\begin{array}{ll}0 & I \\ \Delta & 0\end{array}\right), J\left(\begin{array}{l}\phi \\ \dot{\phi}\end{array}\right)=-\alpha\left(\begin{array}{c}0 \\ \phi^{p}\end{array}\right), V(\phi, \dot{\phi})=\frac{\alpha}{p+1} \int \phi^{p+1} d x$. We take $\mathbb{H}=H^{1} \times L_{2}$ where $H^{1}$ is the Sobolev space. In dimension 2 all the assumptions are satisfied for any $p, \alpha$ while in dimension 3 we require $p=2,3$ or 4 (see Segal [4]). Thus we conclude that Eq. (3) has under these conditions, unique global solutions if $\phi, \dot{\phi}$ is initially small enough. If $\alpha>0$ and $p$ is odd the initial data need not be small because $V \geqq 0$ in that case.

This method can also be applied to coupled systems for which the linearized energy is positive definite. 


\section{References}

1. Chadam,J.M.: Asymptotics for $\square u=m^{2} u+G$. - Global,I.: Existence and decay. Bull. Am. Math. Soc. 76, 1032-5 (1970).

2. Chernoff, P., Marsden, J.: Hamiltonian systems and quantum mechanics (in preparation).

3. Fischer, A., Marsden, J.: The existence of complete spacetimes (in preparation).

4. Segal,I.: Non-linear semi-groups. Ann. Math. 78, 339-364 (1963).

Dr. J. Marsden

Dept. of Mathematics

University of California

Berkeley, Calif. 94720/USA 